

\section{PERCEPCIÓN DE LOS EMPRESARIOS DE UN MUNICIPIO DEL ESTADO DE GUANAJUATO ACERCA DE LAS COMPRAS POTENCIALES Y DE LAS VENTAS REALES.}

\section{PERCEPTION OF ENTREPRENEURS IN A MUNICIPALITY IN THE STATE OF GUANAJUATO ABOUT POTENTIAL PURCHASES AND ACTUAL SALES.}

\section{Resumen}

El presente artículo de investigación hace referencia a algunas estrategias que se deben desarrollar para persuadir al cliente y que en lugar de que sea una compra potencial se concluya en una venta real, entre estas prácticas se encuentra; definir correctamente el mercado meta, diseñar un proceso de ventas, conocer las necesidades, anhelos o deseos del cliente, ofrecer un buen servicio y atención y tener una adecuada mezcla de la mercadotecnia (producto, precio, plaza y promoción).

Palabras clave: Ventas, estrategias, cliente, compra potencial, persuasión.

\section{Abstract}

This research article refers to some strategies that must be developed to persuade the client and that in the place where a potential purchase is concluded in a real sale, among these practices is; Correctly define the target market, design a sales process, know the customer and have a suitable marketing mix (product, price, place and promotion).

Key words: Sales, strategies, client, potential purchase, persuasión.
María de Jesús Soto Vázquez sotovazquezmaria24@gmail.com

María Alejandra Ortega León aortega@upgto.edu.mx

\section{DESCRIPCIÓN DEL PROBLEMA}

La venta es la actividad principal de una empresa, sin ella no tendría futuro por lo que no duraría en el mercado.

En ocasiones es fácil pensar que el cliente necesita el producto 0 servicio y que está dispuesto a encontrarlo pero hoy en día eso pasa a segundo plano, ya que la competencia directa entre las marcas es cada vez más notoria pues en ocasiones los clientes son fieles y se resisten a probar productos nuevos. Por ello es necesario elaborar estrategias para lograr convencer a los clientes de que adquieran los productos y/o servicios.

Para Escalera (2014) generalmente, el cierre de una venta es, al mismo tiempo, el punto difícil y el más importante del proceso de venta que va desde la prospección, la conversión a cliente e identificación de oportunidades y -por supuesto- la venta. 


\section{MARCO TEÓRICO}

Mejía (2012), los seres humanos comenzaron a acumular excedentes de producción por lo que nace el trueque, era una forma de intercambio bastante primitiva en la cual cada participante entregaba parte del producto de su trabajo, a cambio de una parte del producto del trabajo de otro participante.

La American Marketing Asociation, define la venta como "el proceso personal o impersonal por el que el vendedor comprueba, activa y satisface las necesidades del comprador para el mutvo y continuo beneficio de ambos (Thompson, 2005).

También se puede decir que la venta es ceder la propiedad de un producto a cambio de una compensación en dinero, servicio o especies (Andersen, 1997).

Según Kotler (2001), el concepto de venta es otra forma de acceso al mercado para muchas empresas, cuyo objetivo, es vender lo que hacen en lugar de hacer lo que el mercado desea.

Gluck (2016), una estrategia de ventas consiste en un plan que posicione la marca de una empresa o producto para obtener una ventaja competitiva. Las estrategias exitosas que ayudan a la fuerza de ventas se centran en los clientes del mercado objetivo y se comunican con ellos de maneras relevantes y significativas.

Madurga (2015), en el desarrollo de este tipo de estrategias se deben incluir:

$\diamond$ Canales de venta.

$\diamond$ Medios por los que se va a conseguir cada objetivo.

$\diamond$ Tiempo dedicado al desarrollo de cada acción.

$\diamond$ Herramientas de análisis y corrección

Para Jefkins (1997), Ia clave de las ventas está en vender beneficios y no características. Es importante analizar su producto o servicio para determinar cómo puede proporcionar soluciones a los clientes. Los clientes usualmente buscan en sus negocios soluciones para sus problemas. En tal sentido, existen tres elementos fundamentales que los clientes requieren en sus negocios y es hacia donde se deben dirigir los esfuerzos de ventas. Estos elementos son:

$\diamond$ Reducir: bajar costos en general;

$\diamond$ Mejorar: 0 incrementar la eficiencia de lo que ya se tiene;

$\diamond$ Mantener: si no se puede mejorar, se debe mantener lo que se tiene.

Existen varios factores que evitan que el consumidor se convenza de comprar el producto, por ejemplo: las características del producto, la promoción que se le da, el precio, el canal de venta, etc., por eso es importante que como vendedor logre influir en el comportamiento del consumidor puede ser un proceso extremadamente difícil, pero es crucial para mejorar los resultados de las ventas Muñoz (2018).

Para dichos factores, los elementos de la mezcla de la mercadotecnia son los siguientes:

El producto es un conjunto de atributos tangibles e intangibles, que incluyen el envase, el color el precio, la calidad y la marca, más los servicios y la reputación del vendedor y que puede ser un bien tangible, un servicio, un lugar una persona o una idea (Stanton, 1992).

Para Kotler y Armstrong (2012), el precio es la suma de los valores que los consumidores dan a cambio de los beneficios de tener 0 usar el producto 0 servicio.

No obstante es fundamental señalar que el consumidor relacionará el precio con el valor, así podemos establecer dos polos opuestos, un producto gratuito puede carecer de valor, y por otro lado un producto muy caro puede resultar muy valioso (García, 2009).

La distribución es el puente entre la producción y el consumo de los productos y es un puente establecido entre estas actividades (Alegre, 2000).

A través de estos canales conseguimos ampliar nuestro mercado. Al contar con más canales de distribución se estará ampliando nuestra capacidad de alcance a otros mercados 0 , en otras palabras, estaremos aumentando el número de clientes potenciales de los productos o servicios que ofrece la empresa (López, 2019).

La promoción sirve para informar, persuadir y recordarle a un mercado sobre cierto producto o la organización que lo vende, con la esperanza de influir en los sentimientos, creencias o comportamientos de los receptores de la acción promotora. (Stanton, Etzel y Walker 2007). $\diamond$ Presupuesto destinado a cada canal y cada acción de venta.

$\diamond$ Tipo de acciones a realizar, frecuencia y orden en el tiempo. 
Mesa (2013), establece que la Corporación de Desarrollo de Pequeños Negocios del gobierno de Australia menciona que la promoción se refiere a "incentivos a corto término 0 actividades que alienten la adquisición 0 venta de un producto 0 un servicio".

Newman (2017), en su libro "El lenguaje Hipnótico: Mejora tus destrezas de persuasión en ventas y tu habilidad para obtener lo que deseas ante cualquier situación" destaca que existen dos formas para persuadir:

- Lograr que el público objetivo visualice el atractivo de lo que gana con la adquisición del producto, y mostrar el panorama negativo que se generaría al no contar con el producto o servicio (Peker, 2017).

- Es importante mencionar que para que la venta se concluya es necesario seguir un proceso; es la sucesión de pasos que una empresa realiza desde el momento en que intenta captar la atención de un potencial cliente hasta que la transacción final se lleva a cabo, es decir, hasta que se consigue una venta efectiva del producto o servicio de la compañía (Valdés, 2019).

De acuerdo con Fariña (2015), el proceso de ventas se puede dividir en 6 etapas:

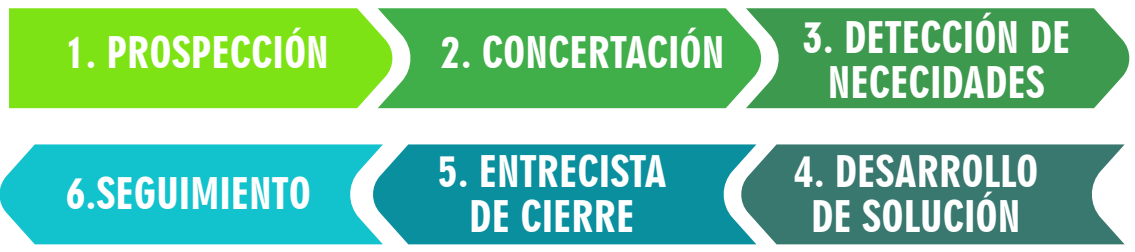

Silva (2020), por otro lado, es importante mencionar que existen 2 tipos de clientes; reales y potenciales y es muy importante enfocarse en ellos para lograr la venta del producto y/o servicio. Un cliente potencial o prospecto en ventas, es aquel que podría convertirse en consumidor y el cliente real es el que ya ha comprado y sigue consumiendo el producto (Pérez 2011).

Una vez que la persona ya se volvió cliente, es necesario fidelizarlo, es decir, retener a los clientes ganados, para que continúen comprando gracias a las experiencias positivas (Escamilla, 2020).

\section{MÉTODO DE INVESTIGACIÓN}

El enfoque bajo el cual se desarrolló este proyecto fue cualitativo y documental, con el objetivo de comprender la percepción para lograr culminar una venta.

\section{RESULTADOS O DISCUSIÓN}

Actualmente, todos los productos de la competencia tienen muy buena calidad y ofrecen los mismos beneficios al cliente, por lo que cada vez es más difícil convencer al consumidor de que adquiera nuestros productos, por ello es mejor diseñar un plan estratégico convincente para lograr cerrar la venta.

Algunas estrategias para lograr una venta son:

$\diamond$ Definir el mercado meta

Para competir en un mercado completo, lo adecuado será centrarse en un sector concreto que permita obtener mejores resultados.

$\diamond$ Diseñar el proceso de ventas dependiendo el producto o giro.

$\diamond$ Estudiar al cliente potencial.

El cliente potencial se interesa, pero aún no se decide a comprar o acudir al servicio de la empresa 0 institución.

$\diamond$ Conocer las expectativas que tienen al comprar.

Es importante conocer lo que esperan los clientes y encontrar la forma sencilla de hacerlo posible de forma diferente y mejor que los competidores.

$\diamond$ Evitar mentir 0 exagerar con el producto u ofertas.

$\diamond$ Prepararse para generar una negociación. 
Saber qué es lo que se está dispuesto a negociar, actitudes, aptitudes e intereses de la otra parte, punto de flexibilidad, etc.

$\diamond$ Dar mayores facilidades para cobrar.

$\diamond$ Ampliar la variedad de productos.

$\diamond$ Ventas personalizadas

$\diamond$ Escuchar y poner atención.

$\diamond$ Darle seguimiento a la venta

$\diamond$ Contar con un sistema de quejas y sugerencias (SQS). $\diamond$ Dar mejores opciones de entrega.

$\diamond$ Buscar más clientes

$\diamond$ Servicio y atención al cliente

$\diamond$ Demostrar porque es la mejor opción de compra

$\diamond$ Responder todas las preguntas que el cliente llegue a tener.

$\checkmark$ Generar más canales de comunicación de tal forma que el cliente tenga alternativas para elegir.

\section{CONCLUSIÓN}

Las ventas y su conexión con la mercadotecnia son de vital importancia para el desarrollo empresarial y, en particular, para que una empresa pueda surgir en el competitivo mundo de los negocios, hoy en día es muy difícil que una nueva marca sea aceptada y mejor aún, preferida por el cliente, pero no es del todo imposible, para lograrlo es necesario enfocarse en que se va a hacer para lograr la atención del cliente y después el convencimiento de la adquisición de la compra.

Derivado del análisis efectuado con las personas entrevistadas, así como de la investigación documental, se considera pertinente seguir las estrategias de ventas pues se lograran mejores resultados, entre las más importantes están:

$\diamond$ Adecuada mezcla de la mercadotecnia: producto, precio, plaza y promoción.

$\diamond$ Contar con un proceso para la pre y post venta.

$\diamond$ Escuchar y poner atención al cliente, de esta forma se conocen sus necesidades, expectativas, percepción y retroalimentación.

$\diamond$ Servicio y atención al cliente eficiente. 


\section{REFERENCIAS}

Alegre, Luis. (2000). Fundamentos de Economía de Empresa: perspectiva funcional, Barcelona, segunda edición, Ariel Económica Editores.

Arthur Andersen, 1997, Diccionario Espasa: Economía y Negocios, Madrid, Editorial Espasa Calpe.

Del libro: El Marketing Según Kotler, de Philip Kotler, Editorial Paidós SAICF, Pág. 136.

Del libro: "Fundamentos de Marketing", Decimocuarta Edición, de Stanton J. William, Etzel J. Michael, Walker J. Bruce, McGraw-Hill Interamericana, 2007, Pág. 506.

Escamilla, G. (2020). Proceso de compra del consumidor. Recuperado de: https://www.rdstation.com/mx/blog/proceso-decompra/

Escalera, J. (2014). La importancia del cierre de ventas. Recuperado de: https://www.liderempresarial.com/la-importanciadel-cierre-de-ventas/

Fariña, A. (2015). EL PROCESO DE VENTAS: LAS 6 ETAPAS Y CÓMO VENDEN LOS MEJORES. Recuperado de: http://xn--alejandrofaria2nb.com/las-6-etapas-del-proceso-de-ventas-como-venden-los-mejores/

García, F (2009). El marketing y la importancia de la fijación de precios. Recuperado: https://www.efdeportes.com/efd130/ el-marketing-y-Ia-importancia-de-Ia-fijacion-de-precios.htm

Gluck. S. (s/f). ¿Qué es la estrategia de ventas?. Recuperado de: https://pyme.Iavoztx.com/qu-es-la-estrategia-de-ventas/

López, C. (2019). ¿CÓMO ELIJO UN CANAL Y UNA ESTRATEGIA DE DISTRIBUCIÓN PARA MI EMPRESA? Recuperado de: https:// www.lainnovacionnecesaria.com/la-importancia-de-los-canales-de-distribucion/

Madurga. J. (2015). Estrategias de venta: ¿cómo superar a tu competencia? Recuperado de: https://www.google.com/amp/s/ es.semrush.com/blog/estrategia-ventas-superar-competencia/amp/

Mejía. M. (s/f). Historia de las ventas. Recuperado de: https://mercadeocreativo.wordpress.com/el-vendedor-exitoso/ historia-de-Ias-ventas/Peker. P. (2017). El sutil encanto de la persuasión en las ventas: sufre menos y disfruta más. Recuperado de: https://www.salesup.com/crm-online/cc-persuasion-en-ventas-estrategia.shtml

Pérez. J. (2011). Definición de cliente potencial. Recuperado de: https://definicion.de/cliente-potencial/

Silva. D. (2020). ¿Qué es un cliente potencial? Recuperado de: https://www.zendesk.com.mx/blog/que-es-cliente-potencial/

Thompson, I. (s/f). Definición de venta. Recuperado de: https://www.promonegocios.net/mercadotecnia/ definicion-concepto-venta.htm\#: : :text = Definici\%C3\%B3n\%20de\%20Venta\%2C\%20Seg\%C3\%BAn\%20Diversos\%20 Autores $\% 3 A$,comprador) $\% 22 \% 20 \% 5 B 1 \% 5 D$.

Valdés, P. (2019). Proceso de venta: ¿Qué es y cómo funciona? Recuperado de: https://www.inboundcycle.com/proceso-deventa-que-es-y-como-funciona 


\section{Derechos de Autor (2021) María de Jesús Soto Vázquez y María Alejandra Ortega León}

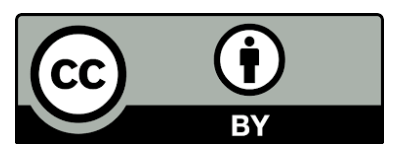

Este texto está protegido por la licencia Creative Commons 4.0.

Usted es libre de Compartir - copiar y redidtribuir el material en cualquier medio o formato- y Adaptar el documento -remezcla, transformar y crear a partir del material- para cualquier propósito, incluso para fines comerciales, siempre que cumpla la condición de:

Atribución: Usted debe dar crédito a la obra original de manera adecuada, proporcionar un enlace a la licencia, e indicar si se han realizado cambios. Puede hacerlo en cualquier forma razonable, pero no de forma tal que sugiera que tiene el apoyo del licenciante o lo recibe por el uso que hace de la obra.

Resumenendelicencia - Textocompretodelalicencia 